\title{
Molecular-level functionalization of electrode surfaces
}

\author{
TADASHI WATANABE \\ Institute of Industrial Science, University of Tokyo, Roppongi, Minato-ku, Tokyo \\ 106, Japan
}

\begin{abstract}
An ultra-thin film of functional molecule(s) can be deposited on an electrode, which then serves as a device to amperometrically monitor compounds of biological interest. The methods of surface functionalization, mostly on tin oxide $\left(\mathrm{SnO}_{2}\right)$ electrodes, include: chemical modification (covalent bond formation between the surface $\mathrm{OH}$ group with a functional group of a molecule); the Langmuir-Blodgett technique, where the $\mathrm{NH}_{2}$ group of a long-chain alkylamine is bound to an enzyme via a small dialdehyde molecule as spacer, and electropolymerization of pyrrole in an electrolyte containing a single enzyme or a sequentially operating bienzyme system. The results of such investigations by the author's group are presented.
\end{abstract}

Keywords. Electrochemical sensor; surface modification; redox enzyme; polypyrrole.

\section{Introduction}

The high performance (substrate selectivity, remarkable acceleration of a specific reaction in aqueous solutions under ordinary temperature and pressure) of biological functional molecules has recently attracted the attention of many workers, and one of the fields of application is the design and development of electrochemical biosensors. The general concept of a biosensor is illustrated in figure 1. A substrate is recognized and processed by a receptor (enzyme, organelle etc.), and this process gives rise to changes of physical parameters (potential, current, etc.) at the electrode-solution interface. The resulting electric signal is recorded to calculate the substrate concentration based on a calibration graph.

The first attempt in this direction was published 35 years ago (Clark and Lyons 1962), where an enzyme (glucose oxidase = GOD)-encapsulated membrane was attached to a metal electrode and the $\mathrm{pH}$ change caused by the oxidation of glucose was monitored using a glass electrode. A slightly later study (Updike and Hicks 1967) used a GOD-encapsulated polyacrylamide gel membrane covering a Clark-type oxygen electrode, and monitored the oxygen consumed in the enzymatic reaction. These works paved the way for succeeding developments in the biosensor field.

Since an electrochemical reaction proceeds on a two-dimensional plane, a monomolecular layer of an enzyme should constitute the basis of a sensor reaction. If such a system (ultra-thin reaction medium) operates satisfactorily, it will possess the additional advantages of (1) a rapid sensor response because mass transfer encounters a minimum barrier, (2) the minimum necessary amount of occasionally expensive enzyme molecules, and (3) easier theoretical analysis of the interfacial processes. These considerations have prompted us to fabricate electrodes carrying an ultra-thin enzyme layer, and to examine their performance as biosensing devices. Several results of such investigations are presented and discussed in what follows. 


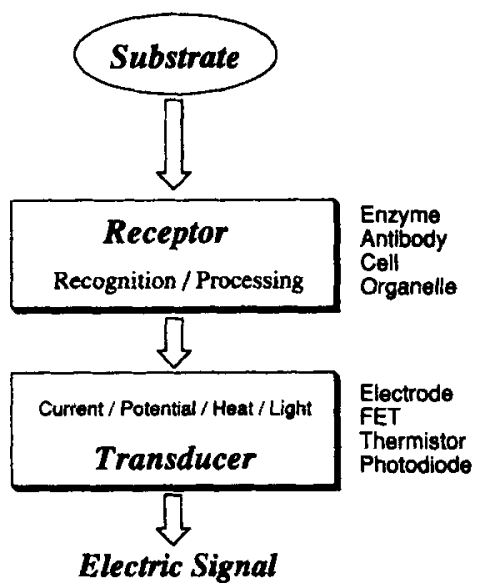

Figure 1. Operational principle of electrochemical biosensors.

\section{Materials and methods}

Electrode: As electrode material, we use tin oxide $\left(\mathrm{SnO}_{2}\right)$ throughout, on the following grounds: (1) the material has sufficient electrical conductivity; (2) it is stable enough both chemically and electrochemically, though a reduction (metallic tin formation) takes place at heavily negative potentials; (3) surface modification (covalent bonding of a functional molecule) is easy via the surface $\mathrm{OH}$ groups; (4) the transparency of the material enables direct, spectrophotometric determination of the colored surface species, especially the heme-containing enzymes; and (5) utilization of the semiconducting property of $\mathrm{SnO}_{2}$ leads to fabrication of FET-mode sensing devices (Okawa et al 1988). When compared with $\mathrm{SnO}_{2}$, conventional metal electrodes possess a general drawback in that even the most stable metal $(\mathrm{Au})$ undergoes various degrees of surface oxidation at positive potentials (Watanbe and Gerischer 1981).

Enzymes: Oxidases (glucose oxidase $=$ GOD, glutamate oxidase $=$ GLOD, lactate oxidase $=$ LOD etc), horseradish peroxidase (HRP), catalase, diaphorase, dehydrogenases and so forth have been used as enzymes. The modes of electrode surface functionalization with these molecules are detailed in $\S 3$.

Sensor performance measurements. The amperometric mode was adopted throughout for sensor performance evaluation. An enzyme-deposited $\mathrm{SnO}_{2}$ plate served as working electrode, an $\mathrm{Ag} / \mathrm{AgCl}$ electrode as reference electrode, and a platinized $\mathrm{Pt}$ as auxiliary electrode. The potential of the enzyme electrode was controlled by a potentiostat. A batch system was used, where the current level change was recorded upon addition of a substrate into the electrolyte solution. In most measurements, the temperature of the test solution was maintained by circulating thermostated water around the electrochemical cell.

\section{Results and discussion}

\subsection{Enzyme monomolecular layer-modified electrodes}

The $\mathrm{SnO}_{2}$ surface was treated first with 3-aminopropyl triethoxysilane (APTES) to yield $\mathrm{O}-\mathrm{Si}$ bondings with free amino groups protruding outward, then reacted with 
glutaraldehyde $(\mathrm{GA}) \mathrm{OHC}-(\mathrm{CH})_{3}-\mathrm{CHO}$ to form a Schiff-base bonding $-\mathrm{N}=\mathrm{CH}-$. The electrode was finally treated with a GOD aqueous solution to give Schiff-base bondings between the $\mathrm{CHO}$ group of GA and the enzyme amino groups. The resulting enzymemodified surface is schematically illustrated in figure 2 (Okawa et al 1989). The ninhydrin assay after hydrolysis of the surface enzyme molecules (for GOD etc.) and the direct spectrophotometric assay of the heme at the Soret absorption peak (for HRP) ensured that the surface population of the enzyme corresponds approximately to a monomolecular layer.

A typical performance of a GOD-modified electrode is shown in figure 3 . The linear dynamic response range covers more than three decades, well encompassing the normal blood glucose level $(3-5 \mathrm{mM})$. In this case, glucose is oxidized by the surface GOD, and the by-product, $\mathrm{H}_{2} \mathrm{O}_{2}$, is oxidatively detected on the $\mathrm{SnO}_{2}$ electrode. Since the working potential is fairly positive (over $+0.7 \mathrm{~V} \mathrm{vs} \mathrm{Ag} / \mathrm{AgCl}$ ), compounds of a reducing nature in biological fluid samples would interfere with the glucose determination. Further, the response was rather slow (of the order of 5-10 min for completion), probably because the two-electron oxidation of $\mathrm{H}_{2} \mathrm{O}_{2}$ is not fast enough on the $\mathrm{SnO}_{2}$ surface. This has led us to devise another mode of surface modification/operation as described below.

\subsection{HRP/GOD heterobilayer-modified electrode}

Horseradish peroxidase (HRP) can reduce $\mathrm{H}_{2} \mathrm{O}_{2}$ at a mild potential of $\approx+0.15 \mathrm{~V}$ vs. $\mathrm{Ag} / \mathrm{AgCl}$. We thus attempted to fabricate an HRP monomolecular-layer modified

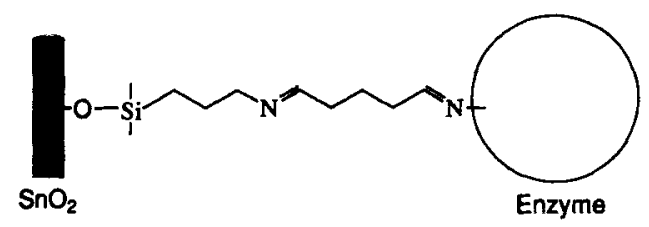

Figure 2. Chemical modification of $\mathrm{SnO}_{2}$ surface with enzyme molecules.

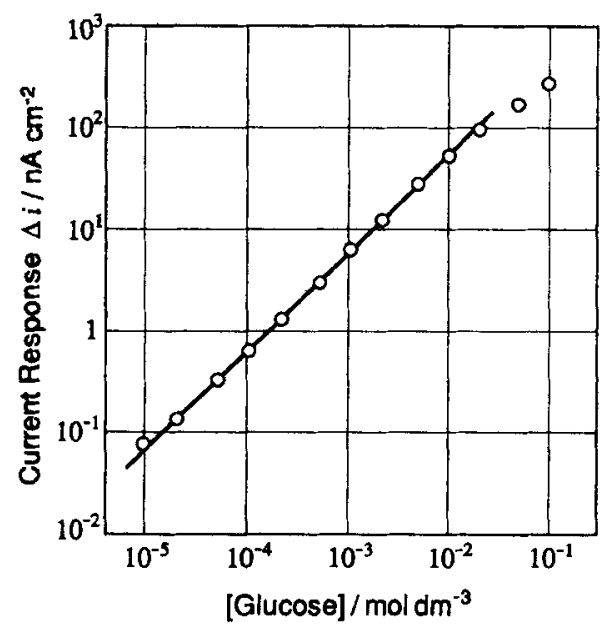

Figure 3. Response characteristics of GOD monolayer-modified $\mathrm{SnO}_{2}$ electrode. 
electrode as an $\mathrm{H}_{2} \mathrm{O}_{2}$ sensor. On this electrode, the detection limit of $\mathrm{H}_{2} \mathrm{O}_{2}$ was found to be as low as $10^{-8} \mathrm{M}$, and the response (cathodic current increase) was complete within about $30 \mathrm{~s}$. Moreover, the reproducibility of the sensor output was excellent, the absolute current levels coinciding with one another to within 5\% for 3-5 independent fabrications and operations.

Based on these pieces of information, we proceeded to prepare an HRP/GOD heterobilayer-modified electrode, first by chemical modification of the $\mathrm{H}_{2} \mathrm{O}_{2}$ surface with HRP in the manner described above, then by treatment of the electrode surface with GA and finally with GOD aqueous solution. A typical result (calibration curve for glucose) is depicted in figure 4 , which compares the sensitivity of the bilayer electrode with those of a GOD monolayer electrode and a GOD crosslinked membrane electrode carrying roughly 200 GOD layers. As is clearly seen, the sensitivity of the bilayer electrode is well comparable to the "thick" membrane electrode, demonstrating the effectiveness of the present surface molecular design. In addition, the response time of the bilayer electrode was 1-2 min, being much faster than for the simple GOD monolayer electrode, as expected (Tatsuma et al 1989).

This strategy has been extended to fabricate lactate, pyruvate, cholesterol, and uric acid sensors by use of HRP/oxidase combinations (Tatsuma and Watanabe 1991a) and, by switching the HRP/oxidase system into diaphorase/dehydrogenase systems, to obtain sensitive electrochemical sensors for glucose, lactate, and ethanol (Tatsuma and Watanabe 1991b).

\subsection{Enzyme model electrode}

Since the substrate normally cannot get into the active center of the enzyme in the $\mathrm{H}_{2} \mathrm{O}_{2}$ sensing by means of natural $\mathrm{HRP}$, one needs to use an appropriate electron mediator (a ferrocene derivative in this case). This causes a problem when applying this type of electrochemical sensor to actual biological samples. In view of this, we examined fabrication of "reagentless" $\mathrm{H}_{2} \mathrm{O}_{2}$ sensors by using a small model molecule, heme nonapeptide (microperoxidase), which is the core part of the natural cytochrome and

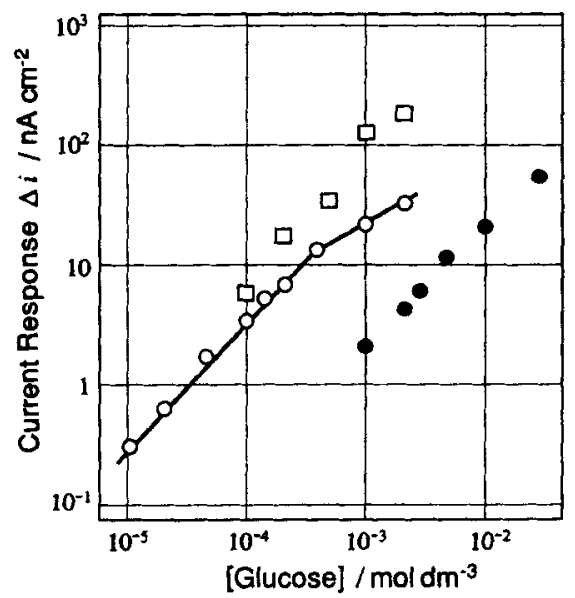

Figure 4. Response characteristics of GOD/HRP heterobilayer-modified electrode $(O)$ as compared with those of GOD monolayer electrode $(\Theta)$ and GOD crosslinked membrane electrode $(\square)$. 
mimics peroxidase activity. The electrode prepared by the chemical modification technique indeed showed a fast response to $\mathrm{H}_{2} \mathrm{O}_{2}$ with a reasonably wide dynamic range (Tatsuma and Watanabe 1991c). Further, the coordination property of a series of nitrogen-containing compounds was explored to develop a novel sensor, by monitoring the decrease in the $\mathrm{H}_{2} \mathrm{O}_{2}$ reduction current upon addition of such nitrogencontaining molecules (Tatsuma and Watanabe 1992a).

As was originally expected, the steady-state and transient responses of these enzyme monolayer-and bilayer-modified electrodes were satisfactorily analyzed on the basis of simple diffusion (mass transfer) and electron transfer models (Tatsuma and Watanabe $1992 \mathrm{~b}, \mathrm{c})$.

\subsection{Enzyme Langmuir-Blodgett film electrodes}

By depositing first a stearic acid/stearylamine mixed monolayer by means of the Langmuir-Blodgett (LB) technique on the $\mathrm{SnO}_{2}$ electrode surface that had been rendered hydrophobic, and then treating the surface with a GOD aqueous solution, the surface was covered with roughly a monomolecular layer of GOD. This responded well to glucose, with sufficient substrate specificity as shown in table 1 . When a small fraction of the fatty acid layer was replaced with a stearyl derivative of ferrocene, the ferrocene site acted as a catalyst for the $\mathrm{H}_{2} \mathrm{O}_{2}$ oxidation, and the current response was nearly doubled (Tatsuma et al 1991).

\subsection{Enzyme encapsulation into ultra-thin polypyrrole films}

Pyrrole can be polymerized by anodic oxidation on an electrode, and coexistence of an enzyme in the electrolyte solution would yield an enzyme-encapsulated polypyrrole (PPy) film. The average thickness of the PPy film can be easily controlled by the electropolymerization charge, giving a film of $\approx 100 \AA$ thickness at $5 \mathrm{mC} \mathrm{cm}^{-2}$ for example. Furthermore, the PPy is known to be electroconductive, so that direct electron exchange may take place between the enzyme and the electrode, and hence we need not use electron mediators for the sensor operation. We attempted to utilize these features to fabricate high-performance electrochemical biosensors carrying a stable, ultra-thin enzyme layer.

A typical result for an HRP/PPy electrode (Tatsuma et al 1992) is illustrated in figure 5. Roughly four decades of dynamic range are attained, with the sensitivity (current level) increasing with an increase in the polymerization charge up to about $5 \mathrm{mC} \mathrm{cm}^{-2}$ (effective thickness $100 \AA$ as mentioned above). A further increase in the thickness tends to lower the sensitivity, probably because of the slowing down of mass transfer across the membrane. Note that the thickness of $100 \AA$ corresponds to only

Table 1. Substrate specificity of GOD LB membrane electrode to $5 \mathrm{mM}$ sugars.

\begin{tabular}{lc}
\hline Sugar & Relative response \\
\hline D-Glucose & 100 \\
D-Galactose & 0 \\
D-Fructose & $0 \cdot 3$ \\
D-Mannose & $0 \cdot 8$ \\
L-Glucose & 0 \\
Sucrose & $0 \cdot 3$ \\
\hline
\end{tabular}




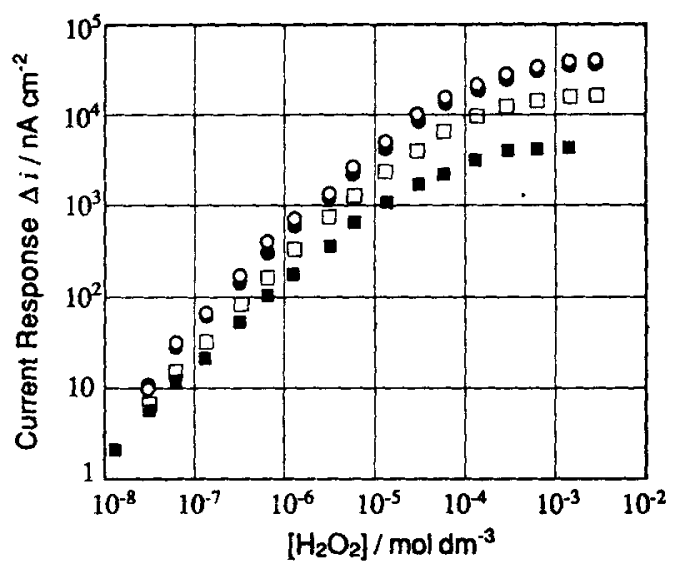

Figure 5. Response characteristics of HRP/PPy electrodes prepared with electropolymerization charge of $0.5(\square), 1.0(\square), 5.0(O)$ and $10(0) \mathrm{mC} \mathrm{cm}^{-2}$.

a few effective molecular layers of the enzyme even when the enzyme molecules are incorporated in a tightly packed state, since the diameter of HRP is about $20-30 \AA$.

\subsection{Double enzyme incorporation into polypyrrole}

Detection of glucose (and other substrates) by use of an oxidase/HRP bienzyme system, as mentioned above for the heterobilayer surface modification, was attempted for the PPy electrodes also. In this case pyrrole is electropolymerized in an electrolyte solution containing the two enzymes in question, with an optimized molar ratio. A typical result for a $100-\AA$ thick GOD/HRP/PPy is given in figure 6(Tatsuma et al 1993a, b). Here an experimental point represents an average of four independent fabrication/operation runs yielding a standard deviation as small as $5 \%$. Compared with the GOD/HRP heterobilayer-modified electrode (figure 4), the sensitivity is improved by a factor of 20 to 30. As far as the glucose determination is concerned, the most prominent feature of this electrode is that an operational potential as low as $+0.15 \mathrm{~V} \mathrm{vs}$. $\mathrm{Ag} / \mathrm{AgCl}$ can be used, and hence many of the reducing compounds (ascorbic acid for instance) coexisting in biological fluids will not interfere with the determination. This system is therefore one of the best ones for amperometric detection of a substrate for which an oxidase is available. The electron mediation process within the enzyme-encapsulated PPy layer has been analyzed theoretically (Tatsuma and Watanabe 1993).

This fabrication mode has recently been extended to sensing of an amino acid, L-glutamate, by using GLOD (glutamate oxidase)/HRP bienzyme system incorporated in PPy, with satisfactory results (Yoshida et al 1995).

\subsection{Extension of linear response range to higher concentrations: Substrate-purging enzyme electrodes}

While the HRP/PPy electrode exhibits a low detection limit, general high sensitivity and wide dynamic range (figure 4), one drawback is that the response tends to be saturated at $\mathrm{H}_{2} \mathrm{O}_{2}$ concentrations above around $10^{-4} \mathrm{M}$. Though such a feature is inherent in an enzymatic process, extension of the linearly responding range to higher concentrations is occasionally desired for practical applications. For this purpose, we 


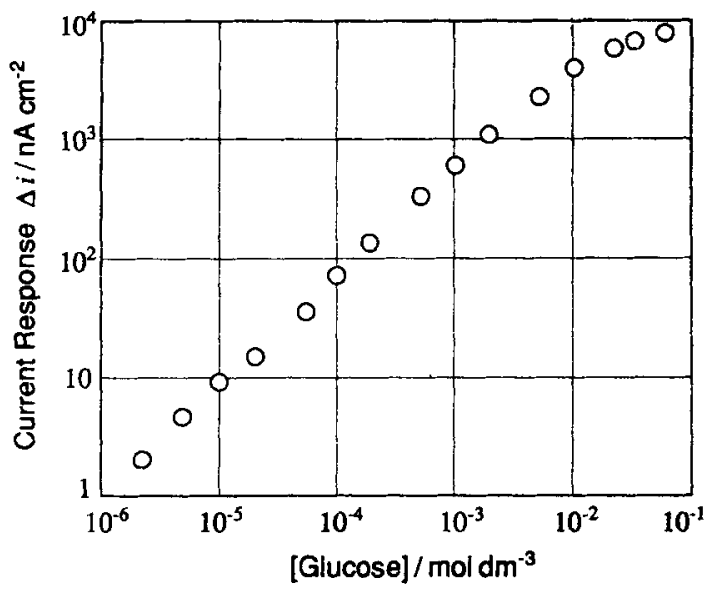

Figure 6. Response characteristics of GOD/HRP/PPy electrode at $+0.15 \mathrm{~V}$ vs. $\mathrm{Ag} / \mathrm{AgCl}$.

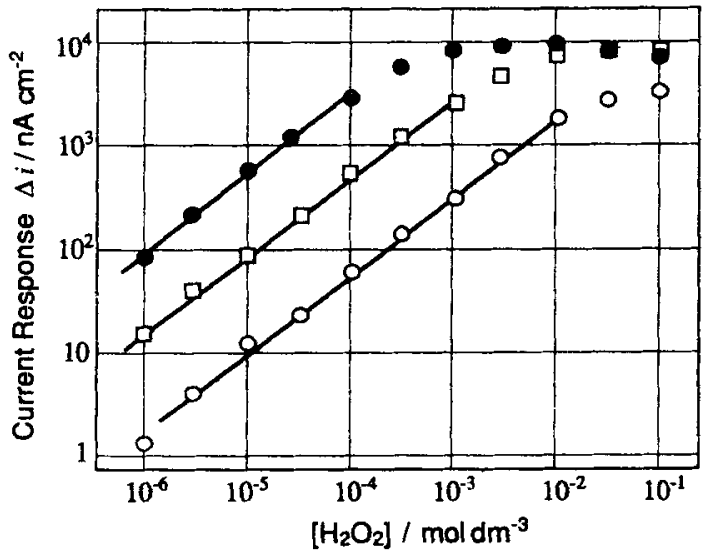

Figure 7. Response characteristics of CAT (catalase)/BSA-HRP/PPy electrodes at $+0.17 \mathrm{~V}$ vs. $\mathrm{Ag} / \mathrm{AgCl}$. PPy electropolymerization charge, $5 \mathrm{mC} \mathrm{cm}^{-2} ; \mathrm{CAT}$ content in BSA layer, $0(O), 2(\square)$, and $20(O) w t \%$.

designed a "substrate-purging" electrode, by coating an HRP/PPy layer with a catalase-encapsulated BSA (bovine serum albumin) thin film (Tatsuma et al 1994). Since catalase consumes $\mathrm{H}_{2} \mathrm{O}_{2}$ partially during passage through the BSA layer, its effective concentration on the HRP/PPy surface would be lowered to a range where a linear response is expected. The result displayed in figure 7 , showing a two-decade extension of the dynamic range though sacrificing the sensitivity roughly 50 -fold, indeed demonstrates the validity of the present design.

\section{Acknowledgments}

The author is grateful to Dr T Tatsuma, Tokyo University of Agriculture and Technology, Dr S Yoshida, and other colleagues for their significant contributions to the biosensor development project in his laboratory. 


\section{References}

Clark L C Jr and Lyons C 1962 Ann. N.Y. Acad. Sci. 10229

Okawa Y, Sukigara M, Yoshida S and Watanabe T 1988 Bull. Chem. Soc. Jpn. 611179

Okawa Y, Tsuzuki H, Yoshida S and Watanabe T 1989 Anal. Sci. 5507

Tatsuma T and Watanabe T 1991a Anal. Chim. Acta 24285

Tatsuma T and Watanabe T 1991b J. Electroanal. Chem. 310149

Tatsuma T and Watanabe T 1991c Anal. Chem. 631580

Tatsuma T and Watanabe T 1992a Anal. Chem. 64143

Tatsuma T and Watanabe T 1992b Anal. Chem. 64625

Tatsuma T and Watanabe T 1992c Anal. Chem. 64630

Tatsuma T and Watanabe T 1993 Anal. Chem. 653129

Tatsuma T, Okawa Y and Watanabe T 1989 Anal. Chem. 612352

Tatsuma T, Tsuzuki H, Okawa Y, Yoshida S and Watanabe T 1991 Thin Solid Films 202145

Tatsuma T, Gondaira M and Watanabe T 1992 Anal. Chem. 641183

Tatsuma T, Watanabe T and Watanabe T 1993a Sensors and Actuators B13-14 752

Tatsuma T, Watanabe T and Watanabe T 1993b J. Electroanal. Chem. 356245

Tatsuma T, Watanabe T, Tatsuma S and Watanabe T 1994 Anal. Chem. 66290

Updike S J and Hicks G P 1967 Nature (London) 214986

Watanabe T and Gerischer H 1981 J. Electroanal. Chem. 117185

Yoshida S, Kanno H and Watanabe T 1995 Anal. Sci. 11251 